\title{
EDITORIAL
}

\section{LA PUBLICACIÓN CONTINUA FRENTE A LA PUBLICACIÓN PERIÓDICA. OTRA VUELTA DE TUERCA EN LA EDICIÓN DE LAS REVISTAS CIENTÍFICAS EN INTERNET}

\section{Cristina Pérez Andrés}

Comité de redacción de la Revista Española de Salud Pública.

Desde que en 1665 vieran la luz las dos primeras revistas científicas, Le Journal des savants en enero ${ }^{1}$ y Philosophical transactions, fundada en marzo $^{2}$, muchos han sido los cambios producidos en la edición científica. Lo raro sería que en 350 años no los hubiera habido pero lo llamativo es que la mayor parte de ellos se han producido en los últimos veinte años, propiciados por el desarrollo por Tim Berners Lee de la Wordl Wide Web (www), red informática mundial ${ }^{3}$ y su presentación pública en abril de $1993^{4}$.

Las revistas científicas empezaron a editarse con el objetivo de publicar resúmenes de los libros que se iban publicando y a partir del siglo XIX el artículo científico pasó a ser el medio de comunicación de los resultados de las investigaciones ${ }^{5}$, multiplicándose continuamente el número de revistas científicas existentes que nacían con carácter periódico, ya fuera semanal, quinquenal, mensual, bimestral...

El desarrollo de la WWW viene siendo tan rápido que las revistas científicas comenzaron su andadura por internet tres o cuatro años más tarde de su presentación, desde mediados de la década de los noventa del pasado siglo XX. Las múltiples consecuencias que ello ha tenido para la edición científica afectan no solo al producto, el artículo publicado, sino también a todo su proceso editorial, sean quienes sean las personas a las que corresponde cada una de sus fases: las que investigan, las que escriben el artículo, las que se encargan del proceso editorial o las que leen los trabajos.

Para la edición electrónica, los cambios comenzaron con el soporte de presentación de los artículos a la redacción de las revistas y de estas a las imprentas, que pasó del papel mecanografiado al archivo electrónico. Primero se grabó en disquete, que seguía enviándose a las redacciones por correo postal, siendo sustituido enseguida por el correo electrónico. En la actualidad la mayoría de las revistas utilizan una plataforma en línea, de las que la más conocida es Open Journal System ${ }^{6}$. Simultáneamente comenzaba la publicación electrónica, al principio coexistiendo con su versión impresa que, poco a poco, se va abandonando. Incluso existen revistas recientes que han nacido con esta forma exclusiva de edición y que en 12 años se han colocado a la cabeza de las más citadas, como es el caso de PlosOne?

Con la edición electrónica y la ventaja del poco espacio que precisa llegó la posibilidad de la recopilación de los trabajos publicados no solo en la propia web de cada revista sino también en diversas bibliotecas virtuales y/o repositorios, ya sean temáticos o institucionales ${ }^{8}$. Quizás haya sido este paso el que más ha contribuido a la generación y al éxito de la iniciativa para el acceso abierto en internet del texto completo de los artículos (open acces) $)^{9-11}$.

La inmediatez de la edición electrónica exigía un cambio más, la publicación de los artículos con los resultados de las investigaciones de 
manera inmediata a su aceptación, lo que termina su proceso editorial. Esto es, la publicación continua frente a la publicación periódica.

La publicación continua lleva a la desaparición de la periodicidad con la que se publican los números, ya sea semanal, quincenal, mensual, bimestral,... quedando el volumen (que suele ser anual) como único compartimento en el que los artículos se incluyen continuamente (rolling volumen). Aunque algunas revistas, sobre todo las que mantienen su edición impresa para los suscriptores, siguen recopilando en números los trabajos publicados de forma continua. Por ejemplo el British Medical Journal (BMJ) lo hace en un número semanal ${ }^{12} \mathrm{yel}$ Journal of the National Cancer Institut (JNCI) en un número mensual ${ }^{13}$. Sin embargo, un estudio con una muy pequeña muestra de editores de revistas científicas (21), observa más satisfacción en las personas responsables de la edición cuando se hace en un volumen sin números que cuando los trabajos se reúnen en números discretos ${ }^{14,15}$. Es muy posible que ello dependa de la cantidad de artículos que publica la revista, resultando más cómodo continuar con los números periódicos si es grande y sin recopilarlos cuando no lo es.

Sea con números periódicos o sin ellos, desde el año 2013 la mayoría de las revistas, tanto a nivel nacional como internacional, han adoptado el modelo de publicación continua ${ }^{12,13,16-20}$.

La publicación continua afecta a la manera de paginar los artículos y, por tanto, a la forma en que son citados en las referencias bibliográficas. Hasta ahora, al comenzar un volumen al principio de cada año, la paginación empezaba en la página 1 de su número 1 y terminaba en el número de página al que hubiera llegado su último número. Con la publicación continua esto cambia, aunque el formato para la nueva paginación está sin estandarizar y de momento cada revista diseña el suyo.

Por poner algunos ejemplos, en BMJ cada artículo en línea tiene un identificador único en lugar de un número de página, el cual debe ser citado en la referencia bibliográfica de todos los artículos en línea y en la edición impresa, así como en PubMed y en los demás índices en los que está indizada ${ }^{12}$.

British Journal of Radiology comenzó la publicación continua a principios de $2013^{17}$ y en las referencias bibliográficas utiliza el número del volumen y un identificador, sin incluir las cifras correspondientes al número ni a las páginas, siendo esta su forma definitiva.

En ambas revistas, el digital objet identifier (DOI) va incluido en el artículo pero no en la referencia bibliográfica.

El Journal of the National Cancer Institut $(\mathrm{JNCI})^{13}$ comenzó la publicación continúa en 2014, manteniendo los números mensuales así como su edición impresa para suscriptores. $\mathrm{Su}$ forma de citar cada trabajo sí incluye el DOI.

En ninguna de las tres revistas existe ya la paginación del artículo en las referencias bibliográficas.

Desde que en 2007 la National Library Of Medicine (NLM) de Estados Unidos publicara la primera edición de Citing Medicine ${ }^{21}$, el Comité Internacional de Editores de Revistas Biomédicas (ICJME por sus siglas en inglés) dirige a esta publicación en sus Recomendaciones para la realización, notificación/información, edición, y publicación de trabajos académicos en las revistas médicas ${ }^{22}$ para conocer cómo referenciar los diferentes tipos de documentos citados en un artículo ${ }^{23}$. En el prólogo de la segunda edición de Citing Medicine, publicada en $2015^{24}$, Joyce Backus se cuestiona si sigue teniendo sentido terminar una cita bibliográfica haciendo referencia a un número periódico y se plantea si es necesario citar dónde se ha publicado un trabajo. Subraya que el nombre de los autores, el título del trabajo y la fecha de publicación siguen siendo necesarios pero, en nombre de la NLM, desea que en un futuro se simplifiquen las referencias bibliográficas de los documentos publicados directamente en internet. Leyendo Citing Medicine se entiende muy bien por qué. Casi hace falta un manual para manejarlo. Sin embargo no he encontrado 
nada en él sobre cómo citar los artículos publicados bajo el modelo de publicación continua, solo lo relativo al formato epubahead que, en principio, es provisional mientras se imprime un trabajo.

Scielo pone como ejemplos las citas sugeridas por dos revistas y por PubMed ${ }^{25}$. La sugerida por PlosOne incluye: Autores (año). Título. Revista volumen (número), número de artículo. DOI. La sugerida por Biomed Central tiene: Título. Autores. Revista año, volumen: número de artículo día mes año. Y la sugerida por PubMed es Autores. Título. Revista. Año mes día;volumen y el DOI. PubMed es el único que no incluye número electrónico de artículo. La referencia elegida se deberá incluir en cada una de las páginas del artículo, que también irán numeradas desde el 1 hasta el número que ocupe la última página.

La Revista Española de Salud Pública viene publicando los trabajos de forma continua con paginación provisional desde marzo de 2013 cuando el proceso editorial de un artículo termina, lo que suele ocurrir entre $0 \mathrm{y}$ 3 días después de haber sido definitivamente aceptado. Este intervalo de días depende de la rapidez con la que los autores corrigen las pruebas de imprenta. Sin esperar a que el número en el que va a quedar incluido esté terminado y pueda tener paginación definitiva, el trabajo cuyo proceso editorial ha terminado se publica en la web de la revista ${ }^{26}$ en el índice de postprints, terminología que lo define como la versión definitiva de un trabajo ya evaluado, aceptado, publicado y, lo que es más importante, que se puede difundir y $\operatorname{citar}^{27}$.

Esta forma provisional de la paginación de los artículos publicados como postprints provocó cambios en el nombre de los ficheros pdf de cada trabajo, que incluía el número de página definitiva por el que comenzaban. El nombre del fichero pdf es la última parte del URL (por las siglas en inglés de Uniform Resource Locators) en la web de la revista. Si la paginación de un trabajo puede ser provisional no conviene en absoluto que lo sea el URL en el que va a estar disponible, el cual debe ser definitivo desde su publicación. Cuando comenzamos la publicación continua el número de página por el que comenzaba el artículo fue sustituido por las iniciales del nombre y dos apellidos de su primer/a autor/a.

El próximo año 2016 el volumen de la Revista será el 90, es decir, la Revista Española de Salud Pública cumple 90 años y los va a empezar inaugurando este nuevo sistema de publicación continua de los artículos en un volumen anual, en lugar de mantener la publicación periódica bimestral que hemos tenido hasta ahora.

Por ello, el próximo volumen 90 modificará su índice cronológico en el sentido de que ya no estará dividido en seis números bimestrales sino en tipos de trabajo ${ }^{28}$, desde el cuál se hará un hipervínculo a cada una de las secciones del actual índice por tipo de trabajo ${ }^{29}$. Es decir, como única numeración se mantiene la del volumen en el que se irán incluyendo a lo largo del año los artículos dentro del tipo de trabajo que se trate: editorial, colaboración especial, original, original breve, editorial, revisión,... Cuando existan números monográficos se indicará en el índice cronológico con la palabra "monográfico".

Por lo tanto este editorial es el último que se publica en un número bimestral de la Revista Española de Salud Pública, en su último número, el 6 del volumen 89.

En cuanto a la forma en que un trabajo debe ser citado se seguirá la sugerencia de PubMed que figura como tercer ejemplo de los que pone Scielo.

Dado que hasta ahora la Revista Española de Salud Pública envía un correo electrónico a través de su lista de distribución cada vez que publica un número, se seguirá enviándolo cada vez que publique un artículo. El alta puede solicitarse desde el inicio de la página web de la revista o por correo electrónico (resp@msssi.es). 


\section{BIBLIOGRAFÍA}

1. Journal des savants. Academie des Inscriptions et des belles letres. Disponible en: http://www.aibl.fr/publications/ periodiques/journal-des-savants/

2. The Royal Society. Philosophical Transactions. Disponible en: https://royalsociety.org/journals/publishing350/historyphilosophical-transactions/

3. Berners-Lee T. Pre-W3C Web and Internet Background. World Wide Web Consortium. Disponible en: http://www. w3.org/2004/Talks/w3c10-HowItAllStarted/?n=15

4. Méndez AM. World Wide Web. Wikipedia. Disponible en: https://es.wikipedia.org/wiki/World_Wide_Web

5. Roca Rosell AM. Sociedades y academias científicas ¿estrategias sociales o elitismo? Quark: Ciencia, medicina, comunicación y cultura.2003; 28-29.

6. Public Knowdledge Project. Open Journal System. Disponible en: https://pkp.sfu.ca/ojs/

\section{PlosOne. Disponible en: http://www.plosone.org/}

8. Pérez Andrés C. La Revista Española de Salud Pública con la edición digital y el acceso abierto en internet. Rev Esp Salud Pública.2011; 84: 551-4. Disponible en: http://www. msssi.gob.es/biblioPublic/publicaciones/recursos_propios/ resp/revista_cdrom/vol86/vol86_6/RS866C_551.pdf

9. Budapest Open Acces Initiative. Disponible en: http:// www.budapestopenaccessinitiative.org/

10. Bethesda Statement on Open Access Publishing. Disponible en: http://www.earlham.edu/ peters/fos/bethesda.htm

11. Berlin Declaration on Open Access to Knowledge in the S c i e n c e s a nd H umanit i es. D is p on ible en: http:// oa.mpg.de/lang/en-uk/berlin-pro-zess/berliner-erklarung

12. Godlee F, Delamothe T, Smith J. Continous publication. BMJ. 2008 Jun 28; 336(7659): 1450. Disponible en: http:// www.bmj.com/content/336/7659/1450

13. Allegra CJ. Continuous Publication of JNCI. JNCI. Disponible en: http://jnci.oxfordjournals.org/content/ early/2013/11/20/jnci.djt351.full

14. Cirasella J, Bowdoin S. Just Roll with It? Rolling Volumes vs. Discrete Issues in Open Access Library and Information Science Journals. J Libr. 2014; 915 Aug 2013

15. Hayman R. Editors View the Continuous Publication Model as a Satisfactory Alternative for Open Access LIS Journals. Evid Based Libr Inf Pract. 2014; 9. Disponible en: http://ejournals.library.ualberta.ca/index.php/EBLIP/article/ view $/ 22165$
16. Estimates of the Continuously Publishing Core in the Scientific Workforce PlosOne. http://journals.plos.org/plosone/article?id=10.1371/journal.pone. 0101698

17. Anderton S, Harvey L. Continuous publication: ready, set, cite! BJR. Disponible en: http://www.ncbi.nlm.nih.gov/pmc/ articles/PMC3664984

18. New Publication Model for Drug Metabolism and Disposition, The Journal of Pharmacology and Experimental Therapeutics, and Molecular Pharmacology. http://www.aspetjournals.org/site/misc/Continuous_Publication.xhtml

19. American Society for microbiology. CMR and MMBR transition to continuous publication model. Disponible en:http://journals.asm.org/site/misc/announcements Feb2015.xhtml

20. Thatje S. Moving forward: change of journal title and continuous article publishing. Naturwissenschaften. 2014; 101:1007-1008 DOI 10.1007/s00114-014-1229-4

21. Citing Medicine. 2007. Capítulo 1.http://www.ncbi.nlm. nih.gov/books/NBK7282/pdf/Bookshelf NBK7282.pdf

22. ICMJE. Recomendaciones para la realización, notificación/información, edición, y publicación de trabajos académicos en las revistas médicas. Disponible en: http://www. icmje.org/recommendations/

23. Sample PubMed Central Citations. Disponible en: http:// www.ncbi.nlm.nih.gov/pmc/pmcdoc/tagging-guidelines/citations/v3/toc.html

24. National Library of Medidne. 2015 Foreword. En: Citing Medicine. Disponible en: http://www.ncbi.nlm.nih.gov/ books/NBK310495/pdf/Bookshelf_NBK310495.pdf

25. Programa SciELO. La publicación continua de artículos en revistas indexadas en SciELO. Scielo. Disponible en: http://www.scielo.org/php/level.php?lang=es\&component=4 $4 \&$ item $=54$

26. Revista Española de salud pública. Disponibleen: www. msc.es/resp

27. Sherpa. Definitions and terms. Disponible en: http://www. sherpa.ac.uk/romeoinfo.html\#prepostprints

28. Revista Española de Salud Pública. Índice cronológico. Disponible en: http://www.msssi.gob.es/biblioPublic/publicaciones/recursos_propios/resp/revista_cdrom/vol75/indices/indice_cronologico_enterol.htm

29. Revista Española de Salud Pública. Índice cronológico. Índice por tipo de trabajo. Disponible en: http://www. msssi.gob.es/biblioPublic/publicaciones/recursos propios/resp/revista_cdrom/vol75/indices/indice_tipo_trabajo.htm 